\title{
Distributed Chloride Prediction System using Neural Network and PIC18F452 Microcontrollers in Water Analysis
}

\author{
S.Kumaravel \\ Department of Computer Science, \\ AVVM Sri Pushpam College \\ (Autonomous), Poondi, Thanjavur, \\ Tamil Nadu, India.
}

\author{
P.Neelamegam \\ Department of Electronics and \\ Instrumentations Engineering, \\ Shanmuga Arts \& Science \\ Technology and Research Academy \\ (SASTRA), Deemed University, \\ Thanjavur, Tamil Nadu, India.
}

\author{
R.Vasumathi \\ Research, Department of Physics, \\ AVVM Sri Pushpam College \\ (Autonomous), Poondi,Thanjavur, \\ Tamil Nadu, India.
}

\begin{abstract}
This paper presents the design of intelligent distributed nodes to predict the chloride concentration of the water samples in neural network environment using PIC18F452 microcontrollers. The nodes are arranged as a coordinate node and four sensor nodes. The training phase of neural network is implemented on coordinate node using Back Propagation Algorithm. The physical parameters of temperature and conductivity of water samples are taken as input parameters and chloride concentration as output parameter for the training phase. The knowledge acquired in the form of weights is stored into all sensor nodes and they are concurrently act as sensing points to predict the chloride concentration by measuring temperature and conductivity using sensors in testing phase. The performance of this scalable system is evaluated using accuracy, speedup and efficiency. The result shows that system attained the linear speed up in analysis of water samples.
\end{abstract}

\section{Keywords}

Distributed System, Artificial Neural Network, Back Propagation Training, Chloride Concentration; Microcontroller

\section{INTRODUCTION}

The water quality and quantity of water resources worldwide is a subject of ongoing concern. The assessment of long-term water quality changes is also a challenging problem. The majority of the chloride ions found in ground water is of marine origin. Seawater can enrich the chloride concentration of ground water in several ways. Elevated Chloride concentration in ground water is the most commonly used indicator of salt-water intrusion in coastal aquifers[1]. Chloride is important in terms of metabolic processes, as it influences osmotic salinity balance and ion exchange. Higher chloride concentrations can reduce the toxicity of nitrite to aquatic life. Since Chloride is a major constitute of saline water, and it directly affects electrical conductivity and temperature[2], the computation of chloride concentration can be found using the correlation of physical parameters of water.

Nowadays a great deal of new demands is made for the sample analysis in water monitoring process at the laboratories, which are situated in regional wise. These demands cannot be fulfilled sufficiently by using conventional chemical methods. The Classical process-based modeling approaches can provide good estimations of water quality parameters, but they usually are too general to be applied directly without a lengthy data calibration process. Limited water quality data and the high cost of water quality monitoring often pose serious problems for process-based modeling approaches. For this reason a new method for fast analysis of water samples is proposed using Artificial Neural Network (ANN) conjunction with Distributed Computing and implemented on microcontrollers. Neural networks attempt to model the brain learning, thinking, storage, and retrieval of information as well as associative recognition. The ANN provides particularly good options, because they are computationally very fast and require may fewer input parameters and input conditions than deterministic models.

The concept of Distributed System has been proved to be an effective way of improving a system operation, which can be extended for the processes that are some what more complex. Benefits by a distributed approach include modularity, improved functionality and performance. Distributed systems allow the deployment and utilization of homogeneous or heterogeneous, network-connected computing resources and offer the potential to analyze, share and manage environmental monitoring information in more flexible and intelligent ways, with a view to making evidence-based decisions and generating new hypotheses on-line [3].

The neural network is a highly potent area to implement the parallel and distributed computing. According to the literature, there are three main kinds of distributed implementation for ANN, High-coupling ANNs, Low coupling ANNs and Data Distributed ANN [3]. In all these methods the distributed-learning method alone is focused to improve the performance of the system. This work is focused the colobration of the Locality based distributed system [4,5] with neural network in testing phase, which offers the good sample handling capabilities and could provide parallel decision support in water diagnosis application with large amount samples. The neural network is trained using back propagation on a node with conductivity and temperature data set as input and its associated known chloride concentration as output. During the training phase, internal weights are iteratively adjusted until the mapping between inputs and output meet some convergence criteria. The weights are then fixed and stored in all sensor nodes. In this system, the trained node can be viewed as a knowledge donor and the sensor nodes are knowledge users. After acquiring the knowledge by all the sensor nodes, which are able to function independently and concurrently to predict the concentration using the sensed physical parameters of water sample.

Towards this direction, this paper investigates the use of distributed methodology for chloride concentration prediction 
scheme that employs Multi Layer Perceptrons (MLPs) using PIC18F452 microcontrollers. In order to handle more number of water samples, this work proposes a way to partition the testing samples across the multiple sensor nodes and evaluate the speed performance of the distributed scheme with respect to a single sensor node implementation.

\section{DESIGN SCHEME OF A DISTRIBUTED MEASUREMENT SYSTEM}

The block diagram of distributed system for computation of chloride concentration in water sample is shown in Figure-1. The ANN based distributed system is a complete system that includes a coordinate node and four sensor nodes. These nodes are connected by using $\mathrm{I}^{2} \mathrm{C}$ bus. The coordinate node is equipped to receive the data set through the keyboard for training. The weight acquired during the trained phase is stored in all the sensor nodes. Each sensor node is able to measure the two physical parameters such as temperature and conductivity in water samples and compute concentration using the stored weight of the trained neural network. The developed system is able to handle four samples concurrently instead of single one.

The PIC18F452 microcontrollers are used in the place of coordinate and sensing nodes. In Figure 1, the Block-A referred a combination of Coordinating Node, Keyboard and LCD. In coordinating microcontroller training of neural network is performed using Back Propagation Algorithm with C code. A Key board is interfaced to give commands and data to coordinate controller. A four row 16 characters alphanumeric display is interfaced to display the information of Input data set, status of the processor, calculated weight values, communication details etc. The Block B, C, D and E represent the Sensor Nodes.

The sensor node arrangement is depicted in Figure-2. The Temperature sensor LM35D, to measure the temperature of the sample is connected to pin 2 (RA0) of microcontroller. The Conductivity cell, to measure the Conductivity of the sample is connected to pin 3 (RA1). The microcontroller PIC18F452 has been found appropriate for the distributed measurement process on the basis of following advantageous features. It is a high performance, enhanced flash microcontroller with 10 bit A/D Converter. The PIC core combines rich instruction set with addressable USART module, which supports RS-485, RS-232 and I2C buses, EEPROM data memory with 10,00,000 erase/write cycles, enhanced Flash program memory with 1,00,000 eraselwrite cycles, flexible timer/counter, a programmable built in multiplexer, three external interrupt pins, master synchronous serial port module and software selectable power saving SLEEP mode. To display the computed results, LCD is connected to Port $\mathrm{D}$ of microcontroller.

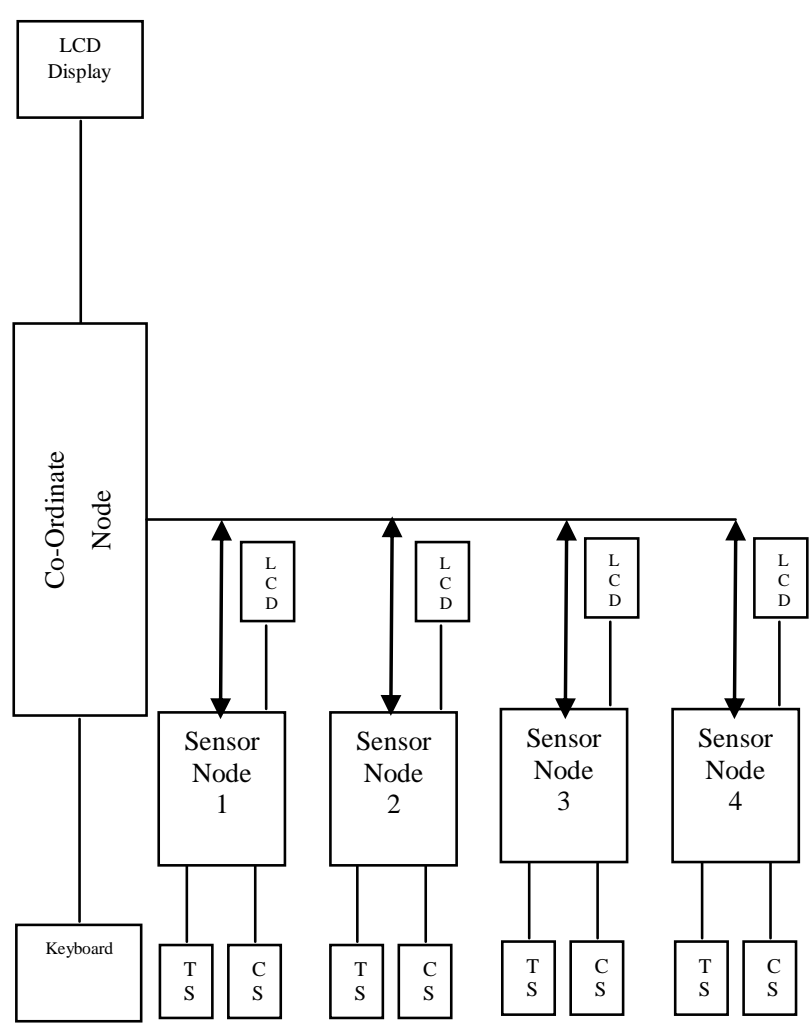

TS: Temperature Sensor CS: Conductivity Sensor

Figure-1: Block Diagram of ANN based Distributed Water Sensing System.

\section{MATERIALS AND METHODS}

The experimental site chosen is located at Manora (Tanjore District) - Mimisal (Pudukkottai District), Middle East coastal region, Tamil Nadu, South India. The sea water samples are collected at every $4 \mathrm{kms}$ from Manora over $100 \mathrm{kms}$ to Mimisal and packed in an air tightens PVC bags and kept in Flask based storage units. To carry out the known input and output for training the Neural Network model, measurements of temperature and electrical conductivity are made In-Situ using Thermometer and conductivity meter (ELICOCM 180) respectively for the samples (Input for the training pattern). The conductivity is reported in terms of micro siemens per centimeter $(\mathrm{mS} / \mathrm{cm})$. The Chloride concentration (output for the training pattern) for the samples is measured using standard method of titration, which uses Titrate solution of silver nitrate $(0.01 \mathrm{~N})$ and indicator of Potassium Chromate with the reddish brown color as end point. 


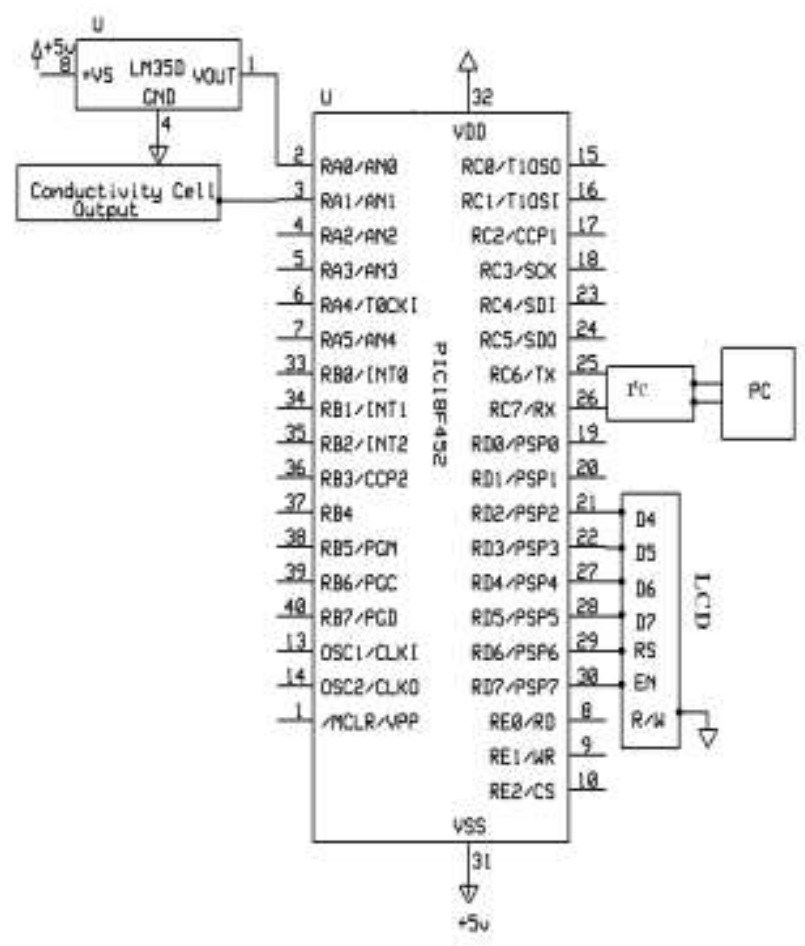

Figure 2: Sensor Node arrangement.

\section{IMPLEMENTATION OF NEURAL NETWORK}

The success of ANNs depends on the architecture, the learning algorithm and its parameters, the transfer function, the number of layers and processing elements (neurons). Back propagation Neural Networks is one of the most common neural networks and they are simple and effective tool. The Architecture of Neural Network with Back Propagation Algorithm consists of Input layer with 2 neurons, hidden layer with 5 neurons and output layer with one neuron as shown in Figure-3.

The learning process, or training, forms the interconnection between neurons and is accomplished by known inputs and output, and presenting these to the ANN in some ordered manner. Due to the interconnection, signals are sent from the input layer to the output layer through the hidden layer. The intensity of the transmitted signal is determined by the weight of the interconnections. It is used to properly obtain the model by iteratively adjusting the values of interconnections between the neurons while the sum of squared residuals between calculated and expected values are minimized. The Back Propagation Algorithm (BPA) uses sigmoid activation function $\mathrm{f}(\mathrm{x})=1 / 1+\mathrm{e}^{-\mathrm{x}}$. The BPA is a supervised learning algorithm that aims at reducing overall system error to a minimum. In this learning procedure, an initial weight vectors $\mathrm{w}_{0}$ is updated,

$$
\mathrm{w}_{\mathrm{i}}(\mathrm{k}+1)=\mathrm{w}_{\mathrm{i}}(\mathrm{k})+\mu\left(\mathrm{T}_{\mathrm{i}}-\mathrm{O}_{\mathrm{i}}\right) \mathrm{f}^{\prime}\left(\mathrm{w}_{\mathrm{i}} \mathrm{x}_{\mathrm{i}}\right) \mathrm{x}_{\mathrm{i}}
$$

Where, $w_{i}=$ The weight matrix associated with $i^{\text {th }}$ neuron; $x_{i}=$ Input of the $i^{\text {th }}$ neuron; $\mathrm{O}_{\mathrm{i}}=$ Actual output of the $\mathrm{i}^{\text {th }}$ neuron; $\mathrm{T}_{\mathrm{i}}=$ Target output of the $i^{\text {th }}$ neuron, and $\mu$ is the learning rate parameter. The specific parameters for learning algorithm are the learning rate $(\mu)$ and momentum $(\alpha)$, which took the values of 0.02 and 0.01 respectively.

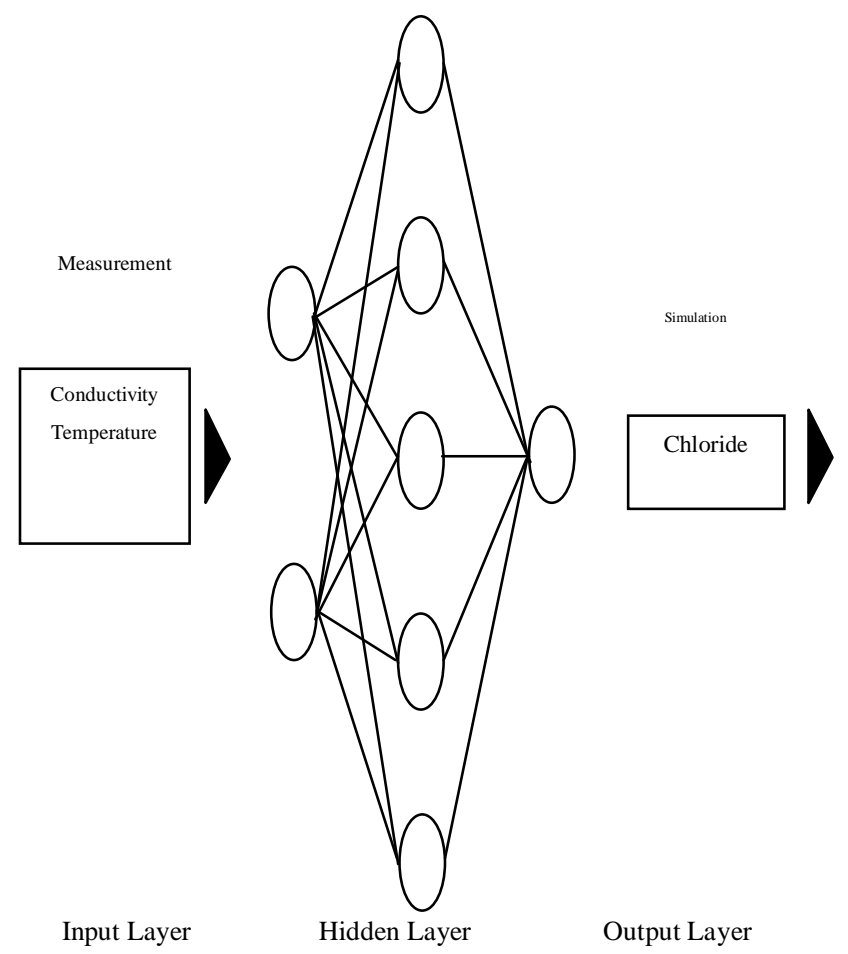

Figure 3: Configuration of the used Multi-Layer Perceptron (MLP). Input layer with 2 neurons, a hidden layer with 5 neurons and an output with 1 neuron.

\subsection{Testing Pattern}

An extensive training phase has to be performed before the testing started. When the training is completed using Back propagation algorithm, the system is tested with the known Input and known output and it is found that the computed output agree with the desired output. The knowledge acquired in the form of weight is stored into all sensor nodes so that the sensor node is able to compute the output (Chloride concentration) of water sample by measuring the temperature and conductivity values. In these phenomena, four sensing nodes are functioning parallel to simulate the chloride concentration. The number of samples to be tested is divided according to the number of sensor nodes. Each divided group of samples is assigned to corresponding sensor nodes. The functioning chart of distributed sensing system is shown in the Figure-4. 


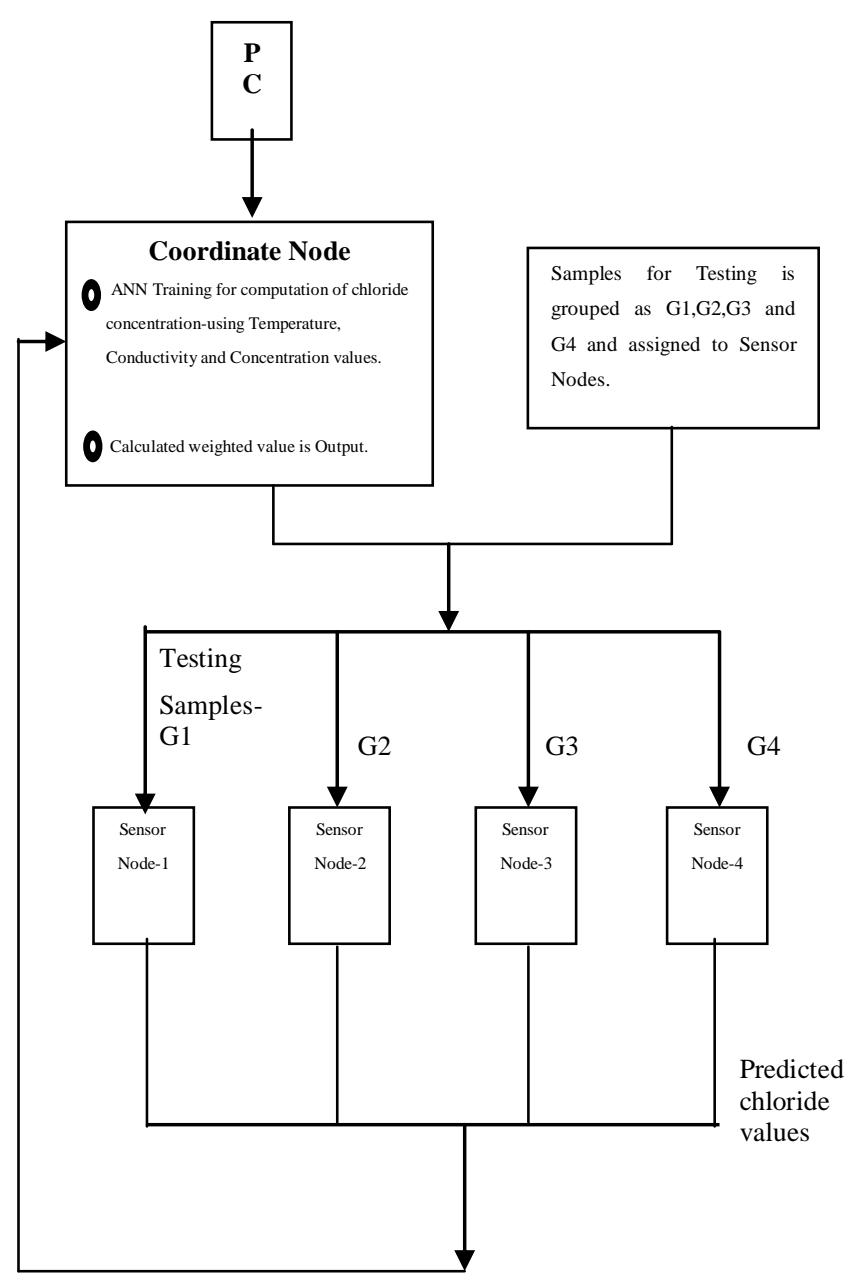

Figure 4: Functioning Chart of Distributed Sensing System.

\section{RESULTS AND DISCUSSION}

The designed distributed system is used for the computation of chloride concentration using the sensor nodes, which measures the conductivity and temperature and computes the concentration of chloride using the trained neural network.

The performance of the proposed system is studied in terms of accuracy, speedup and efficiency. To find the accuracy, the results obtained using the implemented system is compared with that of the conventional method. It is found that the regression equation $(-124.34+1.008 \mathrm{X})$ arrived gives the unity slope and the intercept closes to the zero value which is shown in Figure-5. The Correlation Coefficient $\mathrm{R}=0.99$, shows that the data predicted is well correlated with that of the estimated results. Figure- 6 shows the performance of the neural network for both the outputs for training data and actual value. The speedup and efficiency of the distributed system are obtained by using the ratio between the time required to analyze the set of samples using single sensor node and multiple sensor nodes. The measurement time of single sample consists of sensors activation time, duration of temperature and conductivity measurement, chloride prediction time using Back Propagation Algorithm, and display the result in LCD. The Table-1 described the execution time consumed by the major modules in testing phase of neural network, which is executed on the sensor node. The measurement time of water samples in a sensor node is (MT) is $\sum(a+b+c)$ calculated as $1,07,130$ micro seconds. The potent of the system is exposed by using more number of water samples for that forty water samples are taken into the account to study the performance of the system.

The measurement time of 40 water samples using single sensor node and four parallel sensor nodes is shown in the Figure7 as time space diagram. These two implementations must be comparable so that fair conclusions can be drawn from the measurement results. From the Table-2, the time consumed to predict the chloride concentration for forty water samples using single sensing node (Ts) is 42,85,200 micro seconds, the execution time using 4 sensor nodes (Tp) is 10,71,300 micro seconds. The communication between coordinate node and sensor nodes established in two incidents, first phase is weight distribution from coordinate node to all sensor nodes and the second phase is measured chloride value send by sensor nodes to the co-ordinate node. Let the time required for the first phase is nullified and time consumed in the second phase is calculated as (Tc) is 5783 micro seconds, which is maximum time required to send results by four sensor nodes. The Speedup factor $(\mathrm{Ts} /(\mathrm{Tp}+\mathrm{Tc}))$ of distributed system is 3.9 and the efficiency is (Ts/(Tp*4)*100) is $99.5 \%$. The computation and communication times are calculated using the pin-pong method[10] .

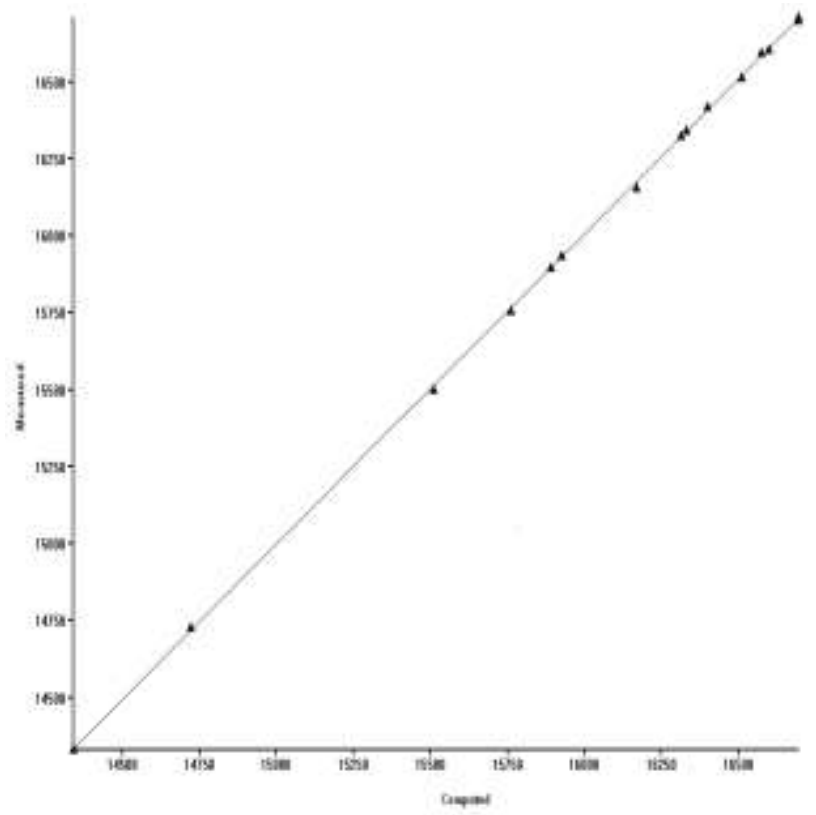

Figure 5. Linear Regression between Measured and Computed Values 


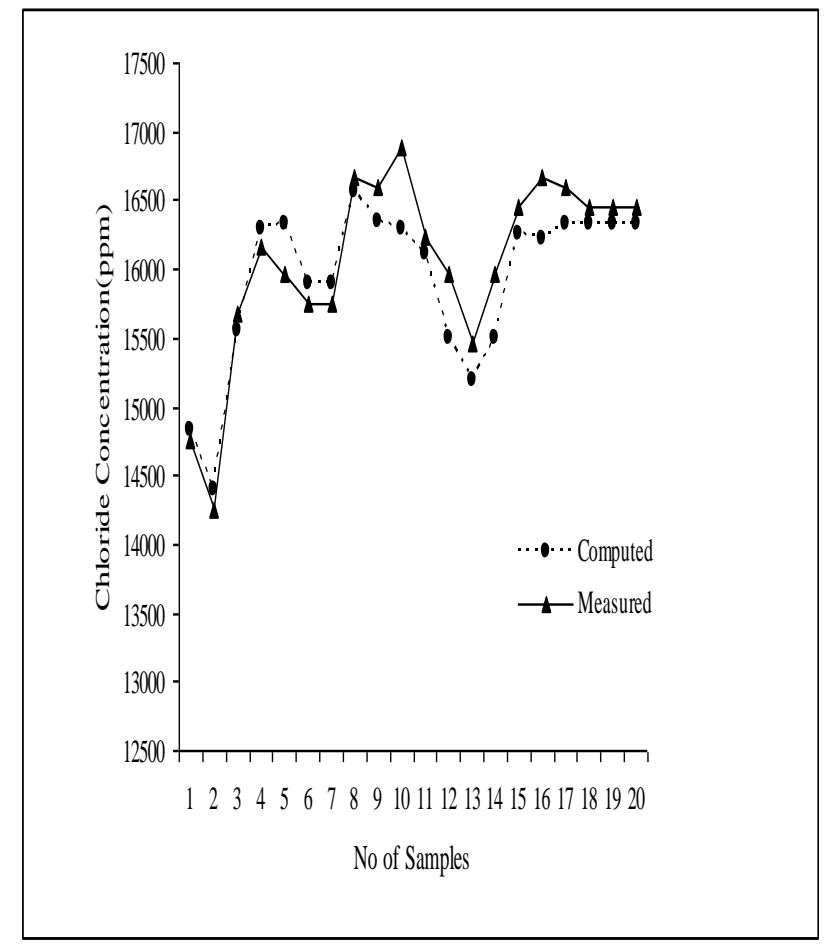

Figure 6: Comparison of Computed and Measured values

Number of Sensor Nodes

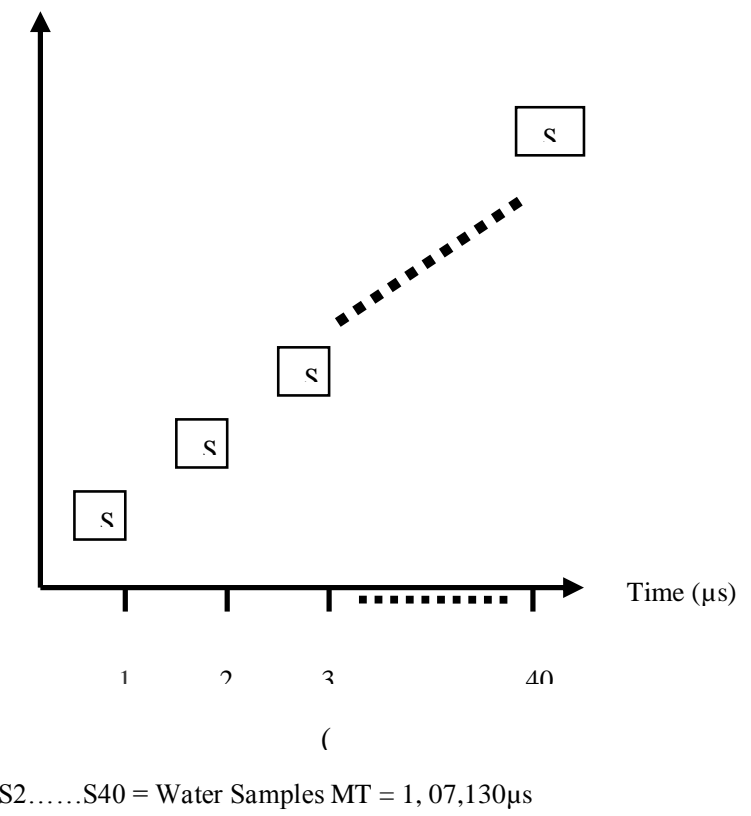

Figure 7. Time Space diagrams for analysis of 40 water samples (a) Time required by single Sensor Node. (b) Time required by four Sensor Nodes.
Table 1 Execution Time of Testing phase modules

\begin{tabular}{|l|c|}
\hline \multicolumn{1}{|c|}{ Module Name } & $\begin{array}{c}\text { Execution Time } \\
(\boldsymbol{\mu s})\end{array}$ \\
\hline a. Temperature measurement & 31195 \\
\hline b. Conductivity measurement & 30935 \\
\hline c. Computation of Chloride Concentration & 45000 \\
\hline
\end{tabular}

Table-2. Performance of the system using $\mathbf{4 0}$ water samples

\begin{tabular}{|l|l|}
\hline \multicolumn{1}{|c|}{ Calculated Items } & Value \\
\hline $\begin{array}{l}\text { Chloride Computation using single sensor Node } \\
\left(\mathrm{T}_{\mathrm{S}}\right)\end{array}$ & $4285200 \mu \mathrm{s}$ \\
\hline $\begin{array}{l}\text { Chloride Computation using four sensor Nodes } \\
\left(\mathrm{T}_{\mathrm{p}}\right)\end{array}$ & $1071300 \mu \mathrm{s}$ \\
\hline Communication overhead $\left(\mathrm{T}_{\mathrm{c}}\right)$ & $5783 \mu \mathrm{s}$ \\
\hline Speed up of the distributed system & 3.9 \\
\hline Efficiency of the system & $99.5 \%$ \\
\hline
\end{tabular}

\section{CONCLUSION}

The distributed measurement system using PIC18F452 microcontroller for the prediction of concentration of sea water samples using Back Propagation Algorithm is implemented. The computation of chloride concentration of sea water samples implemented in distributed environment. The performance based parameters accuracy, speedup and efficiency are evaluated. The accuracy of the system is obtained by a good agreement between the predicted values and experimental values. Further this ANN based prediction system requires no priori-knowledge of the natural physical process of water parameters. If the communications between the coordinate node and sensor nodes are established using wireless technology, the sensor nodes can be used in-situ measurement environment.

\section{REFERENCES}

[1] Duxbury Alison, B., and Alyn, C., Duxbury, 2001.Fundamentals of Oceanography. 270 4th ed. New York: McGraw-Hill.

[2] Cristina Windsor and Rob Mooney, 2008. Verifying the use of specific conductance as 263a surrogate for Chloride in seawater marices. Naples, Florida, USA, June 23-27.

[3] Ianwesley-smith,A parallel artificial neural network implementation, proceedings of the National conference on 
graduate research(NCUR), 2006, University of North Carolina at Asheville, April 2006.

[4] Yang Bo, Wang Xun, research on the performance of the Grid computing for Distributed Neural Network, International journal of Computer Science and Network Security, Volume 6, No 4, April 2006, PP 179-187.

[5] Hardware implementation of distributed Artificial Neural Network for model of Neural functioning, Manuscript Code SP 019.School of Technology, Indiana State University, Terre Haute, Indiana 2005.

[6] P.Neelamegam, S. Kumaravel and R.Ragunathan, Microcontrollers Based Distributed Monitoring System for Fish Water Aquaculture, Instrumentation Science \& Technology, Taylor \& Francis, vol. 36, September 2008, pp.515-524.

[7] Mutlu Avcu, Tulay Yildirim, Microcontroller based Neural Network realization and IRIS plant classifier Application, International XII Turkish Symposium on Artificial and Neural Networks 2003.
[8] Blake Larson, Mahalingam, Stephen, Zhen Zhao, Water Monitoring system for speed and Eramosa Rivers, Proceedings of the Engineering Design III Projects, University of Guelph, 2007, pp 49-50.

[9] F. Cardoba-Montiel, et al, Microcontrollers Hybrid Network for Distributed Instrumentation, Journal of Applied research and Technology, Volume2, No-2, August 2004, pp179-188.

[10] Ali Naiah, Ahemed Elshafie, Othman A.Karim, Prediction of Johor River water quality parameters using Artificial Neural Networks, Europian Journal of Scientific Research, Vol.28. No3, 2009, pp 422-433.

[11] Barry Wilkinson, Michael Allen, Parallel Programming Techniques and Applications Using Networked Workstations and Parallel Computers, Second Edition, Pearson Prentice Hall, 2007. 